\title{
Intracapsular decompression or radical resection followed by Gamma Knife surgery for patients harboring a large vestibular schwannoma
}

\author{
Clinical article
}

\author{
Hung-Chuan Pan, M.D., Ph.D., ${ }^{1,3}$ Jason Sheehan, M.D., Ph.D., ${ }^{2}$ Meei-Ling Sheu, Ph.D., ${ }^{3}$ \\ Wen-Ta Chiu, M.D., P.h.D., ${ }^{4}$ and Dar-Yu YANG, M.D., Ph.D. ${ }^{5}$ \\ ${ }^{1}$ Department of Neurosurgery, Taichung Veterans General Hospital, Taichung, Taiwan; ${ }^{2}$ Department of \\ Neurological Surgery, University of Virginia Health System, Charlottesville, Virginia; ${ }^{3}$ Institute of \\ Biomedical Sciences, National Chung-Hsing University, Taichung ; ${ }^{4}$ Department of Neurosurgery, \\ Taipei Medical University-Shuang Ho Hospital, Taipei Medical University, Taipei; and ${ }^{5}$ Department of \\ Neurosurgery, Chang Bing Show Chwan Memorial Hospital, Changhua, Taiwan
}

\begin{abstract}
Object. Microsurgery is the primary treatment used for patients harboring a large vestibular schwannoma (VS). However, its outcome may lead to hearing impairment and facial nerve dysfunction particularly when resection is extended outside the tumor capsule. When surgery for a large VS consists of intracapsular resection and decompression, better preservation of facial and hearing function are obtained. In this study, the authors compared outcomes of intracapsular decompression followed by Gamma Knife surgery (GKS) with outcomes of standard microsurgery followed by radiosurgery.

Methods. Between August 2003 and October 2008, 35 patients harboring large VSs (> $3 \mathrm{~cm}$ in diameter) were enrolled in this study. Eighteen patients underwent intracapsular decompression followed by GKS (Group I), and 17 patients underwent radical extracapsular resection followed by GKS (Group II). In all cases GKS was performed with a margin dose of $12 \mathrm{~Gy}$. All patients were followed up for at least 3 years. All patients also underwent periodic audiography, electroneuronography (ENoG), MR imaging, and testing with the SF-36 form. The Student t-test and repeated ANOVA were used for statistical analysis.

Results. The mean ages of the patients $( \pm$ SEM) in Groups I and II were $50 \pm 3.0$ and $49 \pm 2.3$ years, respectively. The female/male ratios were 8:10 in Group I and 7:10 in Group II. All patients had excellent facial function as measured according to the House-Brackmann Facial Grading System (Grade I or II) preoperatively. After the operation, 16 patients $(89 \%)$ in Group I retained excellent facial function, whereas only 6 patients (35\%) in Group II had excellent facial function $(\mathrm{p}<0.01)$. In Group I, 11 patients had serviceable hearing, and all $11(100 \%)$ retained hearing function after the operation. In Group II, 11 patients had serviceable hearing, but none retained hearing function postoperatively $(\mathrm{p}<0.001)$. In Group I, the mean tumor volume $( \pm \mathrm{SEM})$ was $17.5 \pm 1.1 \mathrm{~cm}^{3}$, and the postoperative volume was $9.35 \pm 1.02 \mathrm{~cm}^{3}$. In Group II, the mean tumor volume was $16.4 \pm 0.95 \mathrm{~cm}^{3}$, whereas the postoperative volume was $1.1 \pm 0.14 \mathrm{~cm}^{3}(\mathrm{p}<0.001)$. After GKS, the tumor volume was reduced to $5.12 \pm 1.1 \mathrm{~cm}^{3}$ and $0.9 \pm 0.1 \mathrm{~cm}^{3}$ in Groups I and II, respectively. No patients experienced adverse effects after GKS. The mean return-to-work times were $2.4 \pm 0.16$ and $33.4 \pm 4.3$ weeks in Groups I and II, respectively $(\mathrm{p}<0.001)$. According to the results obtained using the 36-Item Short Form Health Survey (SF-36), patients in Group I enjoyed more significant improvements in quality of life than patients in Group II ( $\mathrm{p}<0.001)$.

Conclusions. Intracapsular decompression followed by GKS afforded a better neurological outcome and quality of life than radical extracapsular resection followed by GKS. Further application of this approach in patients harboring large VSs seems warranted.
\end{abstract}

(http://thejns.org/doi/abs/10.3171/2012.6.GKS12697)

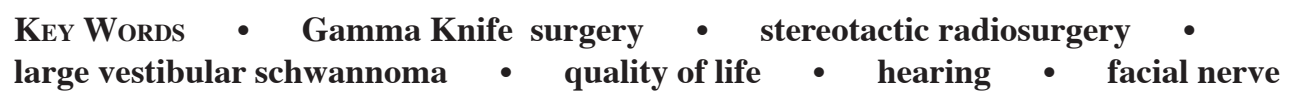

\footnotetext{
Abbreviations used in this paper: $\mathrm{BP}=$ bodily pain; $\mathrm{ENoG}=$ electroneuronography; $\mathrm{GH}=$ general health perceptions; $\mathrm{GKS}=\mathrm{Gamma}$ Knife surgery; $\mathrm{HB}=$ House-Brackmann Facial Grading System; $\mathrm{IAC}=$ internal auditory canal; $\mathrm{MH}=$ mental health; $\mathrm{PF}=$ physical functioning; $\mathrm{RE}=$ role limitations due to emotional problems; RP = role limitations due to physical health; $\mathrm{SF}=$ social functioning; SF-36 = 36-Item Short Form Health Survey; QOL = quality of life; VP = ventriculoperitoneal; VS = vestibular schwannoma; VT $=$ vitality.
}

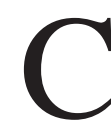
ONTROVERSY exists as to what is the best treatment strategy for patients harboring VSs. Treatment options include watchful waiting, microsurgery, stereotactic radiosurgery, and fractionated stereotactic radiotherapy. ${ }^{21}$ In small-to-medium sized VSs, radiosurgery yields a favorable local tumor control rate and preservation of neurological function, which make radiosurgery an increasingly preferred approach when compared with microsurgery. ${ }^{36,40}$ 
In patients harboring large VSs and, especially, those patients in whom there is also brainstem compression, microsurgery is favored over radiosurgery because the latter approach is unlikely to lead to immediate relief from the mass effect on the brainstem. Nevertheless, in cases of large VSs, the outcome of microsurgery may be compromised by facial and hearing dysfunction. . $^{2,18,19}$ The best treatment approach for dealing with such VSs remains controversial. Proposed strategies include subtotal removal followed by observation or radiosurgery of the residual tumor, and complete removal of the tumor in one or more stages. ${ }^{1,4,16,43,50}$ Whatever surgical approach is taken, preservation of facial nerve function is complicated by significant stretching during surgery and the extreme vulnerability of the nerve. Large VSs represent such a challenging clinical entity that the goal of trying to preserve cranial nerve function while treating these lesions is even questioned by some surgeons. ${ }^{4,5,57}$

When dealing with large VSs, there is a treatment dilemma after subtotal resection of the tumor. If a waitand-scan policy is adopted, the possibility of morbidity and even mortality can arise if the tumor progresses. Alternately, typical complications directly related to radiosurgery are proportional to radiation dose and tumor volume. Radiosurgery can cause VS swelling, ${ }^{10,15}$ which is one reason that this approach is generally avoided in cases of large VSs. Complete microsurgical removal of large VSs is attended by a considerable risk of long-term suboptimal cranial nerve outcomes; for example, good facial nerve function (HB Grade I or II) has been reported in only $27 \%-58 \%$ of patients. . $^{5,18,23,28,51,58}$ Subtotal removal of large vestibular tumors, on the other hand, was reported to preserve excellent facial nerve function and serviceable hearing in nearly $100 \%$ of patients. ${ }^{12,42}$ Previous reports have shown long-term growth control with minimal risk to cranial nerves after GKS for small residual tumors. ${ }^{6,26,35,37-39}$ Thus, the combination of subtotal removal followed by radiosurgery should be a reasonable treatment strategy to preserve cranial nerve function and achieve tumor control.

To date, there have only been a few studies in which outcomes of subtotal removal followed by radiosurgery in the treatment of large VS have been analyzed. .11,16,34,50,55 $^{2}$

The present study was conducted to compare the surgical results obtained by intracapsular decompression followed by GKS with those obtained by radical extracapsular resection followed by GKS. Specifically, we assessed outcomes regarding facial nerve and hearing function as well as QOL.

\section{Methods}

\section{Patient Population}

From August 2003 to October 2008, 38 patients harboring large VSs ( $>3 \mathrm{~cm}$ in diameter) were enrolled in this study. Eighteen patients underwent intracapsular decompression followed by GKS (Group I). Twenty patients underwent radical extracapsular resection of the tumor; however, postoperative MR imaging in 3 of these patients did not reveal any residual tumor. As such, these
3 patients did not undergo postresection GKS and were excluded from this study. One of these 3 patients, who had serviceable hearing prior to treatment, suffered hearing loss after radical resection. One patient with excellent facial function (HB Grade I) retained reasonable facial function (Grade III), and the other two had poor facial function (Grade V) after the operation. The remaining 17 patients who underwent radical resection followed by GKS were included in the current study (Group II) (Fig. 1). The patient characteristics are detailed in Table 1.

Prior to surgery 13 patients -7 in Group I and 6 in Group II-presented with hearing loss, and 1 patient in Group I presented with right facial weakness (HB Grade II). Two patients in Group I and 1 patient in Group II had unsteady gait. Three patients in Group I and 4 patients in Group II presented with severe tinnitus.

The allocation of patients to planned subtotal removal with intracapsular decompression or radical extracapsular resection was determined by patient preference after each patient had been informed about the relative risks associated with the extent of tumor resection and preservation of cranial nerve function with each resection approach. If the patient was more concerned about developing facial nerve palsy than maximizing the extent of tumor removal, intracapsular decompression was performed. If the patient preferred maximum extent of tumor resection to preservation of facial nerve function, the more radical extracapsular resection was performed. In patients who selected intracapsular decompression (Group I), we did not guarantee how much tumor could be resected but only gave an indication based on our surgical experience with releasing brainstem compression. We did not conduct intraoperative MRI and thus could not measure how much residual tumor remained. For this reason, some patients in this study chose radical resection, even if there was a possibility of injury to their facial and hearing function. Informed consent was obtained from patients after the procedures were explained by D.Y.Y. and H.C.P. All radical extracapsular resection of tumors was performed by D.Y.Y. Six of 17 cases of intracapsular decompression were treated by H.C.P.

Before the operation, the patients underwent MRI and completed the SF-36. A hearing test and ENoG were overseen by an otolaryngologist. All microsurgeries were performed using a retrosigmoid approach. The primary goal of surgery was brainstem decompression. In each case, the facial nerve was identified by neurostimulation. For subtotal intracapsular decompression, IAC decompression was not performed. Tumor removal was assisted by using a Cavitational Ultrasonic Surgical Aspirator (Valleylab) and a ring curette. The operation ended when brainstem decompression was observed. For more aggressive, extracapsular resection, we used the Caldwell EMG/EP to monitor facial nerve activity. The seventh cranial nerve was stimulated after its initial exposure, after identification of the medial portion of the nerve, before entering the IAC, and again at various intervals during the operation such as during resection of the tumor in the IAC. During the final dissection, the observation that an evoked potential had disappeared meant the possibility of facial nerve injury and the operation was 


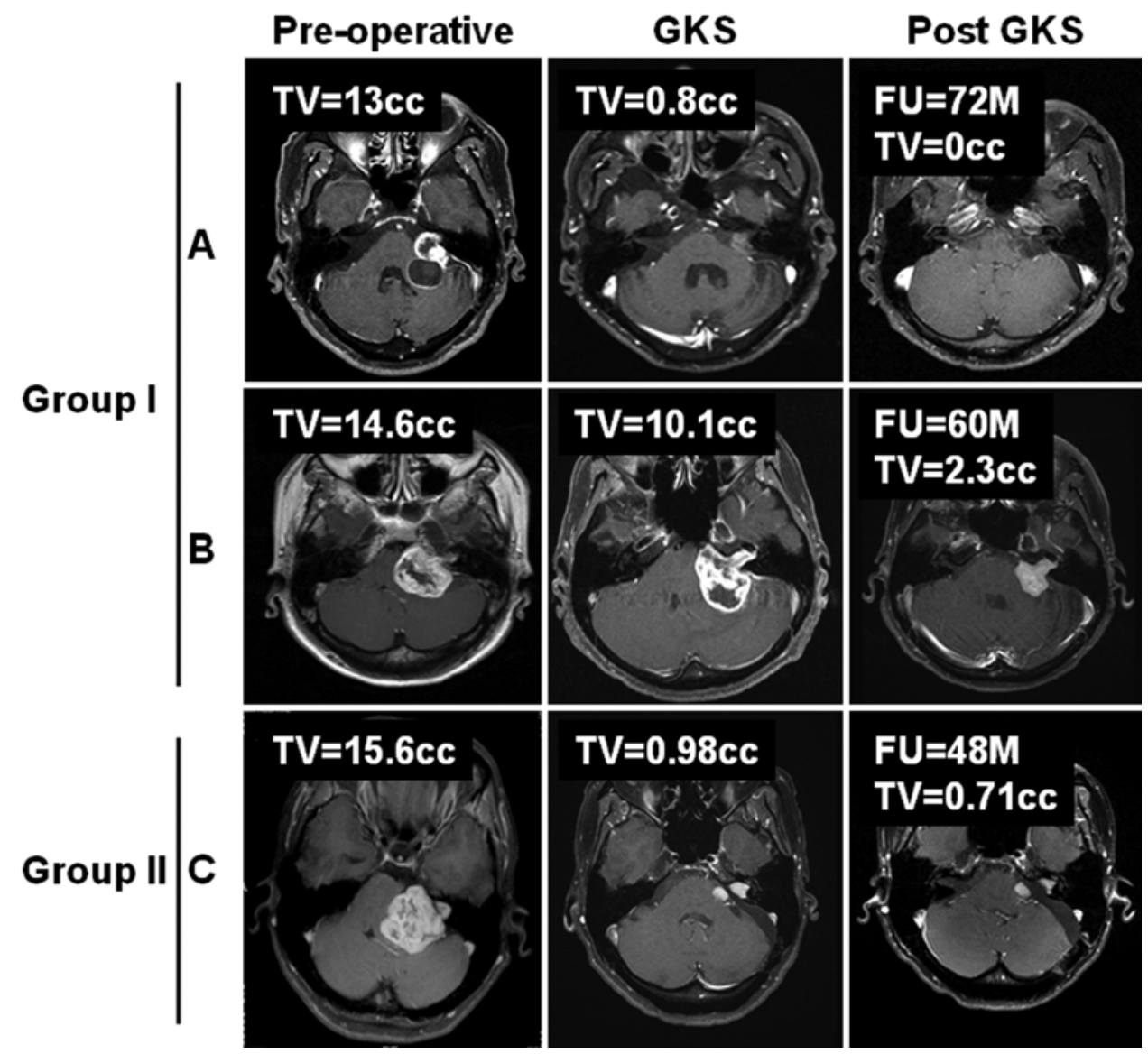

FIG. 1. Contrast-enhanced T1-weighted MR images showing VSs treated by either intracapsular decompression or radical resection followed by GKS. A: Images obtained in a patient who underwent intracapsular resection followed by GKS. The tumor has shrunk substantially. B: Images obtained in a second patient who underwent intracapsular resection followed by GKS. C: Images obtained in a patient who underwent radical extracapsular resection followed by GKS. FU = follow-up; TV = tumor volume.

stopped. All patients received external ventricular drainage for 3-5 days. If the patient suffered facial palsy after the operation, steroid medication was given for 7-14 days postoperatively.

Magnetic resonance imaging was performed 3 months after resection to determine the feasibility of GKS. If there was any abnormal enhancement consistent with a residual tumor, we sent that information to the Taiwan central health bureau to obtain treatment approval, and GKS was then performed.

\section{Gamma Knife Surgical Dose Planning}

Stereotactic radiosurgery was performed using a Leksell Gamma Knife model 4C (Elekta AB). Treatment planning was performed using Leksell GammaPlan software (version 5.3, Elekta $\mathrm{AB}$ ). A dose of 12 Gy was prescribed to the $50 \%$ isodose line, and it covered more than $95 \%$ of the tumor in all treatments.

\section{Clinical Follow-Up}

Patients were followed up at our otolaryngology and neurosurgical outpatient clinics $2-3$ months postradiosurgery. The clinical data obtained at follow-up included the
TABLE 1: Characteristics of patients*

\begin{tabular}{lcc}
\hline \multicolumn{1}{c}{ Characteristic } & Group I (18 patients) & Group II (17 patients) \\
\hline age (yrs) & $50 \pm 3.0$ & $49 \pm 2.3$ \\
F/M ratio & $8: 10$ & $7: 10$ \\
preop tumor vol ( $\left.\mathrm{cm}^{3}\right)$ & $17.5 \pm 1.1$ & $16.4 \pm 0.95$ \\
pre-GKS tumor vol (cm $\left.{ }^{3}\right)$ & $9.35 \pm 1.02$ & $1.1 \pm 0.14$ \\
preop facial function & HB Grade I/II, 17:1 & HB Grade I, 17 \\
preop ENoG & $59.5 \pm 2.1 \%$ & $56.7 \pm 2.3 \%$ \\
no. of patients w/ preop & 11 & 11 \\
$\quad$ hearing function (ser- & & \\
$\quad$ viceable hearing) & & $7 \pm 0.4$ \\
time from op to GKS & $3.6 \pm 0.2$ & \\
$\quad$ (mos) & & \\
no. of patients requiring & 1 & \\
$\quad$ placement of a VP \\
shunt
\end{tabular}

* Mean values are expressed as means \pm SEMs. Patients in Group I underwent intracapsular decompression followed by GKS, and patients in Group II underwent radical extracapsular resection of the tumor followed by GKS. 
results of the SF-36, an audiogram, ENoG images, and a detailed neurological examination.

\section{Assessment of Hearing Function}

Serviceable hearing was defined as a speech repetition threshold or pure tone audiogram value less than 50 $\mathrm{dB}$ and a speech discrimination score of $50 \%$ or greater.

\section{Assessment of Facial Nerve Function With ENoG}

Electroneuronography involves electrical stimulation of the facial nerve at or near the stylomastoid foramen and the subsequent measurement and interpretation of the motor response, as recorded at or near the nasolabial fold. Electroneuronography was performed by an audiologist to evaluate the integrity of the facial nerve. The ENoG test is the only "relatively objective" measure of facial nerve integrity. The ENoG is used to compare the neurophysiological response of the normal (healthy) side to the abnormal (diseased) side in order, and data are presented as the ratio of the nerve ipsilateral to the tumor divided by the nerve on the contralateral side.

\section{House-Brackmann Facial Grading System}

The HB scale was used to approximate the quantity of facial nerve function that the patient had at presentation as well as at each follow-up interval. The HB scale allows us to grossly describe the characteristics and degree of facial nerve motion using a subjective analysis scale, which is easily and reliably applied. The HB scale has 6 grades and each grade is reported as a fraction (for example, $1 / 6=$ Grade I). In the HB scale, Grade I indicates perfectly normal, Grade II indicates slight or mild weakness, Grade III indicates moderate weakness with good (normal) eye closure, Grade IV indicates moderate weakness with no volitional eye closure, Grade V indicates severe weakness, and Grade VI indicates total facial paralysis.

\section{Assessment of Quality of Life}

The SF-36 is a validated instrument used to measure a patient's QOL. ${ }^{33}$ The items in the SF-36 cover eight domains or scales including physical functioning $(\mathrm{PF})$, role limitations due to physical health (RP), body pain (BP), general health perceptions (GH), vitality (VT), social functioning (SF), role limitations due to emotional problems (RE), and mental health (MH). In general, the physical component summary encompasses PF, RP, BP, and $\mathrm{GH}$, whereas the mental component summary includes VT, SF, RE, and MH. The scores on the SF-36 scale range from 0 to 100 , with higher scores implying better condition.

\section{Imaging Follow-Up}

Periodic follow-up neuroimaging (MRI) was performed. Studies were obtained every 6 months for the first 2 years following GKS and then yearly thereafter. The post-GKS MRI studies were performed using a 2to 3-mm slice thickness to obtain T1-weighted images and a 3- to 5-mm slice thickness to obtain T2-weighted and FLAIR sequences. The MRI was performed without adding Gd contrast medium. Tumor volumes were determined on the basis of the MR images by using a picture archiving and communication system and were compared with volumes obtained during GKS planning. We graded the neuroimaging response according to the change in tumor volume as follows: $>20 \%$ volume enlargement $=$ tumor increase; $>20 \%$ volume decrease $=$ tumor shrinkage; and stable tumor $=<20 \%$ volume change. ${ }^{33}$ Postradiosurgical imaging studies were available for all patients.

\section{Statistical Analysis}

The descriptive statistics were computed using standard methods to calculate the mean or median values. The Student $t$-test and repeated ANOVA were used. A p value less than 0.05 was considered statistically significant.

\section{Results}

\section{Clinical and Imaging Outcomes}

The mean age $( \pm$ SEM) in Group I was $50 \pm 3$ years and that in Grade II was $49 \pm 2.3$ years. The female/male ratio was 8:10 in Group I and 7:10 in Group II. Thirty-four of 35 patients had preserved HB Grade I facial function and only 1 patient in Group I had facial nerve function of HB Grade II before resection. Sixteen patients (89\%) in Group I retained excellent facial function (Grade I or II) following intracapsular decompression. However, only 6 patients (35\%) in Group II had excellent facial nerve function following radical extracapsular resection $(\mathrm{p}<0.01)$. The mean ENoG in Group I was $59.5 \% \pm 2.1 \%$ preoperatively and remained at $55.3 \% \pm 3.4 \%$ postoperatively $(\mathrm{p}=0.8)$. The mean preoperative ENoG in Group II was $56.7 \% \pm 2.3 \%$, and it decreased to $10.5 \% \pm 1.3 \%$ postresection $(\mathrm{p}<0.001)$.

In Group I, 11 patients had serviceable hearing and all 11 patients (100\%) retained the same hearing function after resection. In Group II, 11 patients had serviceable hearing and none of them retained serviceable hearing after resection $(\mathrm{p}<0.001)$. One patient in Group I had delayed hydrocephalus and underwent placement of a VP shunt. There was no new development of low cranial palsy after the operation. Only 1 patient in Group II required placement of a permanent VP shunt due to delayed hydrocephalus. One patient in Group II suffered from lower cranial nerve palsy after resection.

In Group I, the mean tumor volume $( \pm$ SEM) was $17.5 \pm 1.1 \mathrm{~cm}^{3}$ before the operation and the postresection volume was $9.35 \pm 1.02 \mathrm{~cm}^{3}(\mathrm{p}<0.01)$. In Group II, the mean tumor volume was $16.4 \pm 0.95 \mathrm{~cm}^{3}$, whereas the postoperative volume was $1.1 \pm 0.14 \mathrm{~cm}^{3}(\mathrm{p}<0.001)$.

The time intervals from resection to GKS were $3.6 \pm$ 0.2 and $7 \pm 0.4$ months in Groups I and II, respectively (p $<0.01$ ), and the post-GKS follow-up periods were $57.7 \pm$ 3.3 and $52.7 \pm 1.8$ months, respectively. After GKS, tumor volumes shrank to $5.12 \pm 1.1 \mathrm{~cm}^{3}$ in Group I and $0.9 \pm 0.1$ $\mathrm{cm}^{3}$ in Group II $(\mathrm{p}<0.001)$, and the volumes remained decreased until the last follow-up. There was no additional deterioration in facial nerve function after GKS. One patient in Group I had transient hearing loss following 


\section{Gamma Knife surgery for large vestibular schwannomas}

GKS, but the patient displayed progressive improvement after steroid treatment. This 45-year-old woman harbored a $15.4-\mathrm{cm}^{3}$ acoustic tumor on the right side with facial nerve function (HB Grade I) and serviceable hearing with a pure tone audiogram of $35 \mathrm{~dB}$. She underwent intracapsular decompression for this tumor. After the operation, preservation of both facial nerve function and hearing were noted. Postoperative MRI showed a residual tumor with a volume of $4.5 \mathrm{~cm}^{3}$. The patient underwent GKS 4 months after the operation; the radiation dose to the margin was 12 Gy at the $50 \%$ isodose line. The patient suffered sudden onset of tinnitus and hearing loss 2 days after GKS. She started methylprednisolone treatment with a dosage of $200 \mathrm{mg}$ every 6 hours for 5 days. The patient progressively recovered hearing function. Two months after GKS, the pure tone audiogram was 40 $\mathrm{dB}$. The patient continued to have serviceable hearing 36 months after GKS. Magnetic resonance imaging showed that the tumor had shrunk to the size of $1.5 \mathrm{~cm}^{3}$.

Following GKS, no patients experienced adverse effects, such as those seen by T2 signal changes within the brainstem on MR images. Further outcomes are summarized in Table 2.

\section{Quality of Life After Treatment}

The mean return-to-work times after resection were $2.4 \pm 0.16$ and $33.4 \pm 4.3$ weeks in Groups I and II, respectively ( $<<0.001)$. In Group I, the mean score for physical functioning $(\mathrm{PF})$ before surgery was $82.2 \pm 13.5$. The score remained stable at $84.2 \pm 1.3$ at 3 months after the operation and at $84.2 \pm 1.2$ following GKS until the last follow-up. In Group II, the mean PF score before surgery was $82.3 \pm 1.5$. The score remained stable at $84.2 \pm 1.3$ at 3 months after the operation, and it was $82.6 \pm 1.4$ following GKS until the last follow-up.

In Group I, the mean score for role limitations due to physical health $(\mathrm{RP})$ before resection was $86.1 \pm 3.6$. The score increased to $93.1 \pm 2.7$ at 3 months after resection and remained at that number after GKS. In Group II, the mean RP score before resection was $83.8 \pm 3.7$. The score decreased to $52.9 \pm 7.7$ ( $\mathrm{p}<0.001) 3$ months after resection and remained stable at $52.9 \pm 7.7$ after GKS. In Group I, the mean score for role limitations due to emotional problems $(\mathrm{RE})$ before surgery was $82.1 \pm 4.4$, and it improved to $98.1 \pm 1.9$ at 3 months after resection. The mean RE score remained stable after GKS with little fluctuation until the last follow-up. In Group II, the mean RE score before surgery was $78.1 \pm 4.9$, and it sharply decreased to $54.5 \pm 6.4$ ( $p<0.001) 3$ months after resection. The mean RE score remained stable after GKS with little fluctuation until the last follow-up. In both groups, the mean preoperative, postoperative, and post-GKS vitality (VT) scores remained stable. In Group I, the mean preoperative, postoperative, and post-GKS mental health (MH) scores only showed slight changes. In Group II, the mean $\mathrm{MH}$ score before resection was $89.4 \pm 1.8$. This score sharply decreased to $49.3 \pm 7.3(\mathrm{p}<0.001) 3$ months after GKS and remained stable until the last follow-up. In Group I, the mean preoperative, postoperative, and postGKS social functioning (SF) scores did not show any significant change. In Group II, the mean SF score before
TABLE 2: Outcomes of combined resection and GKS*

\begin{tabular}{lcc}
\hline Variable & Group I (18 patients) & Group II (17 patients) \\
\hline $\begin{array}{c}\text { follow-up period } \\
\text { (mos) }\end{array}$ & $57.7 \pm 3.3$ & $52.7 \pm 1.8$ \\
$\begin{array}{c}\text { tumor vol after GKS } \\
\left(\mathrm{cm}^{3}\right)\end{array}$ & $5.12 \pm 1.1$ & $0.9 \pm 0.1$ \\
$\begin{array}{c}\text { postop facial func- } \\
\text { tion }\end{array}$ & HB Grade I/II, 16:2 & HB Grade I/II/III/IV, 2:4:3:8 \\
$\begin{array}{c}\text { post-GKS facial } \\
\text { function }\end{array}$ & HB Grade I/II, 16:2 & HB Grade I/II/III/IV, 2:4:4:7 \\
$\begin{array}{c}\text { postop ENoG } \\
\text { post-GKS ENoG }\end{array}$ & $55.3 \pm 3.4 \%$ & $10.5 \pm 1.3 \%$ \\
no. of patients w/ \\
$\begin{array}{l}\text { postop service- } \\
\text { able hearing }\end{array}$
\end{tabular}

* Mean values are expressed as means \pm SEMs.

surgery was $87.5 \pm 3.2$. This score decreased to $40.6 \pm 5.9$ $(\mathrm{p}<0.001) 3$ months after GKS and remained stable until the last follow-up.

In both groups, the mean preoperative, postoperative, and post-GKS scores for bodily pain (BP) remained stable. In Group I, the mean preoperative, postoperative, and post-GKS scores for general health perceptions (GH) showed no appreciable changes. In Group II, the mean preoperative $\mathrm{GH}$ score was $93.8 \pm 1.9$. This score decreased to $52.4 \pm 6.4(\mathrm{p}<0.001) 3$ months after resection and remained stable until the last visit. The detailed results of the SF-36 scores are depicted in Table 3.

At the last follow up, the mean QOL scores for RF ( $p$ $<0.001), \mathrm{RE}$ ( $\mathrm{p}<0.001), \mathrm{MH}$ ( $\mathrm{p}<0.001), \mathrm{SF}(\mathrm{p}<0.001)$, and GH $(\mathrm{p}<0.001)$ were significantly greater in Group I than in Group II.

\section{Discussion}

The general consensus on large VSs has been to perform a resection and thereby facilitate brainstem decompression. But with complete resection, a high incidence of cranial nerve dysfunction has been reported. Hence, an alternative approach has been to perform an intracapsular decompression for the purpose of brainstem decompression while maintaining cranial nerve function. For longterm tumor control, GKS has as its target the residual tumor. In this study, we found that planned intracapsular decompression followed by GKS achieved better preservation of seventh and eighth cranial nerve function than more radical extracapsular resection followed by GKS.

For microsurgery, it is well known that the size of the VS is the main predictor of preservation of facial nerve anatomy and function. ${ }^{18,43,44,47,51}$ In cases of large $\mathrm{VSs}$, the anatomical integrity of the nerve is preserved in $78 \%-94 \%$ of patients, but good facial nerve function is only preserved in $38 \%-75 \%$ of cases..$^{5,13,23,45,48}$ However, 
H. C. Pan et al.

TABLE 3: Outcomes on the SF-36 after resection and GKS*

\begin{tabular}{|c|c|c|c|c|c|c|}
\hline \multirow[b]{2}{*}{ Domain } & \multicolumn{2}{|c|}{ Preop } & \multicolumn{2}{|c|}{ Postresection } & \multicolumn{2}{|c|}{ Post-GKS } \\
\hline & Group I & Group II & Group I & Group II & Group I & Group II \\
\hline PF & $82.2 \pm 13.5$ & $83.3 \pm 1.5$ & $84.2 \pm 1.3$ & $84.2 \pm 1.3$ & $84.2 \pm 1.2$ & $82.6 \pm 1.4$ \\
\hline $\mathrm{RE}$ & $82.1 \pm 4.4$ & $78.1 \pm 4.9$ & $98.1 \pm 1.9$ & $54.5 \pm 6.4$ & $98.1 \pm 1.9$ & $54.5 \pm 5.4$ \\
\hline VT & $89.4 \pm 1.9$ & $89.4 \pm 1.8$ & $89.4 \pm 1.9$ & $89.4 \pm 1.8$ & $89.4 \pm 1.8$ & $89.3 \pm 1.9$ \\
\hline $\mathrm{BP}$ & $100 \pm 0$ & $100 \pm 0$ & $100 \pm 0$ & $100 \pm 0$ & $100 \pm 0$ & $100 \pm 0$ \\
\hline $\mathrm{GH}$ & $95.5 \pm 1.6$ & $93.8 \pm 1.9$ & $94.2 \pm 1.9$ & $52.4 \pm 6.4$ & $94.2 \pm 1.9$ & $52.4 \pm 6.4$ \\
\hline
\end{tabular}

* Data are presented as means \pm SDs. Abbreviations: $B P=$ body pain; $\mathrm{GH}=$ general health; $\mathrm{MH}=$ mental health; $\mathrm{PF}=$ physical function; $\mathrm{RE}=$ role limitation due to emotional problem; RP = role limitation due to physical health; $\mathrm{SF}=$ social function; $\mathrm{VT}=$ vitality.

in some reports planned subtotal removal of the tumor achieved nearly $100 \%$ preservation of facial nerve and hearing function. ${ }^{16,42}$ In this study, in the planned subtotal intracapsular resection group, preservation of facial nerve function was $89 \%$, but in the planned radical extracapsular resection group, only $36 \%$ of facial nerve function was achieved. For preservation of facial nerve function, intracapsular decompression should be considered an alternative approach for patients harboring large VSs.

The incidence of giant tumor among all cases of VS has been estimated to be $2 \%-15 \%$ in Western countries, ${ }^{15,46}$ but in developing countries such tumors may be harbored by the majority of VS patients..$^{17}$ The clinical presentation of patients with large VSs differs from that of patients with small tumors. In particular, hearing loss has been observed in $96 \%-100 \%$ of these patients. ${ }^{2,54}$ In some large VS series, $40 \%$ to $60 \%$ of serviceable hearing was still preserved preoperatively, but the hearing preservation rate dropped to $0 \%$ to $36.4 \%$ postoperatively. In the radical resection group in our study, $100 \%$ of patients had hearing loss. However, in the intracapsular decompression group, functional hearing was preserved in all patients.

Stereotactic radiosurgery has become the standard treatment for small-to-medium sized VSs. With radiosurgery, satisfactory clinical outcomes have been reported in patients after long-term follow-up, and radiosurgery typically affords tumor shrinkage, facial nerve preservation, and functional hearing preservation in the majority of patients. ${ }^{6,22,36,39,40}$ However, transient tumor expansion has been observed at 6-12 months postradiosurgery. In those patients who experience transient tumor expansion, the tumor exhibits a mean increase of nearly $39 \%$ to $62 \%$ in volume..$^{31,52,57}$ In patients with larger tumors, transient tumor expansion after radiosurgery can cause morbidity. Some anecdotal reports concerning large VSs treated with GKS have shown nearly $90 \%$ tumor control as well as preservation of satisfactory facial nerve and serviceable hearing function. ${ }^{7,50,54}$ However, the incidence of unsteady gait or VP shunt placement in patients with large VSs after radiosurgery has been reported to be nearly $10 \%$, which is significantly higher than that in smallto-medium sized VS, for which such a complication is usually as low as $2 \%{ }^{6,7,22,39,50}$ Thus, it is seems reasonable to debulk larger tumors to a manageable size so as to facilitate safer radiosurgery. In our study, larger tumors were treated by intracapsular decompression to achieve a tumor size more amenable to GKS. In this way, there was no appreciable rate of adverse events after GKS.

Outcomes after VS treatment tend to focus on those associated with the surgical approach, immediate procedural complications, facial and hearing preservation, and mortality. However, from the patient's standpoint, QOL is an important outcome metric, too, even though few studies have assessed this result. The SF-36 has long been used as a reliable tool in QOL assessment. ${ }^{25,32,33}$ The greater the number of symptoms, such as balance problems, headache, and facial and hearing dysfunction experienced by patients with VSs, the lower the overall SF-36 score and the specific component scores within it. ${ }^{29}$ The SF-36 scores in physical ability, social functioning, emotional status, and vitality were lower after resection of a large VS. ${ }^{32}$ Several studies have consistently demonstrated that facial paresis after surgery will result in lower QOL scores. ${ }^{24,27}$ In the present study, Group I, in which patients underwent intracapsular decompression, showed that significant preservation of facial function can contribute to a significant improvement in QOL.

Regarding working capacity, patients who harbored a large tumor prior to surgery returned to work significantly later than patients who had undergone surgery for a small tumor. ${ }^{32,49}$ An individual's capacity for working and the time interval before returning to work depend on the person's status of neurological function and the length of rehabilitation required for recovery. In Group I, in which patients underwent intracapsular decompression, most patients resumed their preoperative work schedule within 1 month, even with the planned addition of GKS to target the residual tumor. In Group II, in which patients underwent radical extracapsular resection, however, a high incidence of facial and hearing dysfunction was observed; these patients spent more time in rehabilitation and also required facial nerve anastomosis. Based on the length of time before patients could return to work, intracapsular decompression followed by GKS appears to be the optimal approach for patients harboring large VSs. 
Many tests of facial nerve function have been used, including the Hilger test, electromyography, acoustic reflex test, ${ }^{8}$ evoked accelerometry, antidromic nerve potentials, MRI and CT evaluations, maximal and minimal nerve stimulation tests, transcranial magnetic stimulation, ${ }^{41}$ and blink reflex tests..$^{20}$ The most commonly used facial nerve grading scale is the HB scale. ${ }^{14}$ This scale is used to approximate the quantity of volitional motion patients have, based on their clinical facial presentation. The scale is based on clinical observation, and variation among observers exists. The ENoG is an established and reliable method for assessing neural degeneration in various conditions affecting the facial nerve; it allows a subclinical analytical method that can impact medical or surgical decisions. ${ }^{3}$ In this study, we used ENoG as an adjuvant test to assess facial nerve function, and we found that it and the HB scale were highly correlated. Compared with radical resection followed by GKS, planned intracapsular resection followed by GKS afforded superior facial nerve function at the last follow-up examination, when nerve function was assessed using either ENoG or HB methods.

There are several limitations to this study. First, the allocation of patients to the intracapsular compression group or the radical extracapsular resection group was based on patients' preference after they had been informed of surgical options and associated risks and benefits. Second, the statistical power of the current study was limited. Also, the short interval between resection and GKS for residual tumors in our study may not have provided sufficient time for visualizing the tumor and yielding optimal radiosurgical targeting, given that postoperative changes were still present. ${ }^{12}$ In addition, a longer follow-up period in both patient groups is required to show that neuroimaging, clinical, and QOL outcomes remain durable. There has been some debate concerning regrowth of residual tumor following GKS during longterm follow-up. Indeed, there have been some reports of progression or malignant transformation of acoustic schwannomas after GKS. This should make one cautious in the interpretation of outcomes of these two approaches.

Preservation of facial and hearing function is the primary goal in the treatment of large acoustic tumors. Only at very experienced centers can surgical outcomes provide excellent preservation of hearing and facial nerve function. At neurosurgical centers without sufficient surgical experience, outcomes of radical resection are not optimal. Alternatively, intracapsular decompression can be performed at almost any neurosurgical institute with minimal risk to facial and hearing function. Adjuvant GKS can then be performed to control the growth of the residual tumor. It seems that the combination of intracapsular decompression followed by GKS is a logical and alternative treatment for large VSs, especially when the concern is preservation of hearing and facial function.

\section{Conclusions}

For those patients harboring large VSs, intracapsular decompression followed by GKS appears to be a better treatment option than radical resection followed by GKS. Cranial nerve preservation and higher QOL scores on the
SF36's RF, RE, MH, SF, and GH domains are superior with a planned approach of limited intracapsular resection followed by GKS.

\section{Disclosure}

This study was supported by grants from Taichung Veterans General Hospital (No. TCVGH-984902B) and the National Science Council (No. NSC-96-2113-M-075A-001-MY2), Taiwan, R.O.C.

Author contributions to the study and manuscript preparation include the following. Conception and design: Yang. Acquisition of data: Yang, Pan, Chiu. Analysis and interpretation of data: all authors. Drafting the article: Pan. Critically revising the article: all authors. Reviewed submitted version of manuscript: all authors. Approved the final version of the manuscript on behalf of all authors: Yang. Statistical analysis: Sheu. Study supervision: Yang.

\section{Acknowledgments}

The authors thank Mrs. Shu-Zhen Lai and Mrs. Mu-Jung Liu for preparation of the manuscript and the Biostatistics Task Force of Taichung Veterans General Hospital for assistance with statistical analysis.

\section{References}

1. Abe T, Izumiyama H, Imaizumi Y, Kobayashi S, Shimazu M, Sasaki K, et al: Staged resection of large hypervascular vestibular schwannomas in young adults. Skull Base 11:199_ 206, 2001

2. Anderson DE, Leonetti J, Wind JJ, Cribari D, Fahey K: Resection of large vestibular schwannomas: facial nerve preservation in the context of surgical approach and patient-assessed outcome. J Neurosurg 102:643-649, 2005

3. Bendet E, Talmi YP, Kronenberg J: Preoperative electroneurography (ENoG) in parotid surgery: assessment of facial nerve outcome and involvement by tumor-a preliminary study. Head Neck 20:124-131, 1998

4. Brackmann DE, Green JD: Translabyrinthine approach for acoustic tumor removal. Otolaryngol Clin North Am 25: 311-329, 1992

5. Briggs RJ, Luxford WM, Atkins JS Jr, Hitselberger WE: Translabyrinthine removal of large acoustic neuromas. Neurosurgery 34:785-791, 1994

6. Chung WY, Liu KD, Shiau CY, Wu HM, Wang LW, Guo WY, et al: Gamma knife surgery for vestibular schwannoma: 10year experience of 195 cases. J Neurosurg 102 Suppl:87-96, 2005

7. Chung WY, Pan DH, Lee CC, Wu HM, Liu KD, Yen YS, et al: Large vestibular schwannomas treated by Gamma Knife surgery: long-term outcomes. J Neurosurg 113 Suppl:112-121, 2010

8. de Bisschop G, Sarabian A, de Bisschop E, Sarabian N, Zanaret M: [Selection of electrophysiological investigations for diagnosis in idiopathic facial palsy. Twenty years experience in an ENT department.] Rev Laryngol Otol Rhinol (Bord) 119:75-85, $1998(\mathrm{Fr})$

9. Di Maio S, Malebranche AD, Westerberg B, Akagami R: Hearing preservation after microsurgical resection of large vestibular schwannomas. Neurosurgery 68:632-640, 2011

10. Flickinger JC, Kondziolka D, Niranjan A, Maitz A, Voynov G, Lunsford LD: Acoustic neuroma radiosurgery with marginal tumor doses of 12 to 13 Gy. Int J Radiat Oncol Biol Phys 60: 225-230, 2004

11. Fuentes S, Arkha Y, Pech-Gourg G, Grisoli F, Dufour H, Régis $\mathrm{J}$ : Management of large vestibular schwannomas by combined surgical resection and gamma knife radiosurgery. Prog Neurol Surg 21:79-82, 2008 
12. Godefroy WP, van der Mey AG, de Bruine FT, Hoekstra ER, Malessy MJ: Surgery for large vestibular schwannoma: residual tumor and outcome. Otol Neurotol 30:629-634, 2009

13. Gormley WB, Sekhar LN, Wright DC, Kamerer D, Schessel D: Acoustic neuromas: results of current surgical management. Neurosurgery 41:50-60, 1997

14. House JW, Brackmann DE: Facial nerve grading system. Otolaryngol Head Neck Surg 93:146-147, 1985

15. Ito K, Shin M, Matsuzaki M, Sugasawa K, Sasaki T: Risk factors for neurological complications after acoustic neurinoma radiosurgery: refinement from further experiences. Int J Radiat Oncol Biol Phys 48:75-80, 2000

16. Iwai Y, Yamanaka K, Ishiguro T: Surgery combined with radiosurgery of large acoustic neuromas. Surg Neurol 59:283291, 2003

17. Jain VK, Mehrotra N, Sahu RN, Behari S, Banerji D, Chhabra DK: Surgery of vestibular schwannomas: an institutional experience. Neurol India 53:41-45, 2005

18. Jung S, Kang SS, Kim TS, Kim HJ, Jeong SK, Kim SC, et al: Current surgical results of retrosigmoid approach in extralarge vestibular schwannomas. Surg Neurol 53:370-378, 2000

19. Kanzaki J, Tos M, Sanna M, Moffat DA, Monsell EM, Berliner KI: New and modified reporting systems from the consensus meeting on systems for reporting results in vestibular schwannoma. Otol Neurotol 24:642-649, 2003

20. Kimura M, Nakagawa I, Niinai H, Hamada H, Sakai A, Yasuuji M: [Evaluation with blink reflex of bilateral facial palsy.] Masui 49:159-162, 2000 (Jpn)

21. Kondziolka D, Lunsford LD, Flickinger JC: Comparison of management options for patients with acoustic neuromas. Neurosurg Focus 14(5):e1, 2003

22. Kondziolka D, Lunsford LD, McLaughlin MR, Flickinger JC: Long-term outcomes after radiosurgery for acoustic neuromas. N Engl J Med 339:1426-1433, 1998

23. Lanman TH, Brackmann DE, Hitselberger WE, Subin B: Report of 190 consecutive cases of large acoustic tumors (vestibular schwannoma) removed via the translabyrinthine approach. J Neurosurg 90:617-623, 1999

24. Lee J, Fung K, Lownie SP, Parnes LS: Assessing impairment and disability of facial paralysis in patients with vestibular schwannoma. Arch Otolaryngol Head Neck Surg 133:5660, 2007

25. Leplège A, Ecosse E, Verdier A, Perneger TV: The French SF-36 Health Survey: translation, cultural adaptation and preliminary psychometric evaluation. J Clin Epidemiol 51: 1013-1023, 1998

26. Lunsford LD, Niranjan A, Flickinger JC, Maitz A, Kondziolka D: Radiosurgery of vestibular schwannomas: summary of experience in 829 cases. J Neurosurg 102 Suppl:195-199, 2005

27. Magliulo G, Zardo F, Damico R, Varacalli S, Forino M: Acoustic neuroma: postoperative quality of life. J Otolaryngol 29:344-347, 2000

28. Mamikoglu B, Wiet RJ, Esquivel CR: Translabyrinthine approach for the management of large and giant vestibular schwannomas. Otol Neurotol 23:224-227, 2002

29. Martin HC, Sethi J, Lang D, Neil-Dwyer G, Lutman ME, Yardley L: Patient-assessed outcomes after excision of acoustic neuroma: postoperative symptoms and quality of life. $\mathbf{J}$ Neurosurg 94:211-216, 2001

30. Myrseth E, Møller P, Pedersen PH, Lund-Johansen M: Vestibular schwannoma: surgery or gamma knife radiosurgery? A prospective, nonrandomized study. Neurosurgery 64:654663, 2009

31. Nagano O, Serizawa T, Higuchi Y, Matsuda S, Sato M, Yamakami I, et al: Tumor shrinkage of vestibular schwannomas after Gamma Knife surgery: results after more than 5 years of follow-up. Clinical article. J Neurosurg 113 Suppl:122-127, 2010
32. Nicoucar K, Momjian S, Vader JP, De Tribolet N: Surgery for large vestibular schwannomas: how patients and surgeons perceive quality of life. J Neurosurg 105:205-212, 2006

33. Pan HC, Sun MH, Chen CC, Chen CJ, Lee CH, Sheehan J: Neuroimaging and quality-of-life outcomes in patients with brain metastasis and peritumoral edema who undergo Gamma Knife surgery. J Neurosurg 109 Suppl:90-98, 2008

34. Park CK, Jung HW, Kim JE, Son YJ, Paek SH, Kim DG: Therapeutic strategy for large vestibular schwannomas. J Neurooncol 77:167-171, 2006

35. Pollock BE: Vestibular schwannoma management: an evidence-based comparison of stereotactic radiosurgery and microsurgical resection. Prog Neurol Surg 21:222-227, 2008

36. Pollock BE, Driscoll CL, Foote RL, Link MJ, Gorman DA, Bauch CD, et al: Patient outcomes after vestibular schwannoma management: a prospective comparison of microsurgical resection and stereotactic radiosurgery. Neurosurgery 59: 77-85, 2006

37. Pollock BE, Link MJ: Vestibular schwannoma radiosurgery after previous surgical resection or stereotactic radiosurgery. Prog Neurol Surg 21:163-168, 2008

38. Pollock BE, Lunsford LD, Flickinger JC, Clyde BL, Kondziolka D: Vestibular schwannoma management. Part I. Failed microsurgery and the role of delayed stereotactic radiosurgery. J Neurosurg 89:944-948, 1998

39. Prasad D, Steiner M, Steiner L: Gamma surgery for vestibular schwannoma. J Neurosurg 92:745-759, 2000

40. Régis J, Pellet W, Delsanti C, Dufour H, Roche PH, Thomassin JM, et al: Functional outcome after gamma knife surgery or microsurgery for vestibular schwannomas. J Neurosurg 97:1091-1100, 2002

41. Rimpiläinen I, Eskola H, Laippala P, Laranne J, Karma P: Prognostication of Bell's palsy using transcranial magnetic stimulation. Acta Otolaryngol Suppl 529:111-115, 1997

42. Roland JT Jr, Fishman AJ, Golfinos JG, Cohen N, Alexiades G, Jackman AH: Cranial nerve preservation in surgery for large acoustic neuromas. Skull Base 14:85-91, 2004

43. Samii M, Gerganov VM, Samii A: Functional outcome after complete surgical removal of giant vestibular schwannomas. Clinical article. J Neurosurg 112:860-867, 2010

44. Staecker H, Nadol JB Jr, Ojeman R, Ronner S, McKenna MJ: Hearing preservation in acoustic neuroma surgery: middle fossa versus retrosigmoid approach. Am J Otol 21:399-404, 2000

45. Sterkers JM, Morrison GA, Sterkers O, El-Dine MM: Preservation of facial, cochlear, and other nerve functions in acoustic neuroma treatment. Otolaryngol Head Neck Surg 110: 146-155, 1994

46. Tos M, Stangerup SE, Cayé-Thomasen P, Tos T, Thomsen J: What is the real incidence of vestibular schwannoma? Arch Otolaryngol Head Neck Surg 130:216-220, 2004

47. Tos M, Thomsen J: The translabyrinthine approach for the removal of large acoustic neuromas. Arch Otorhinolaryngol 246:292-296, 1989

48. Tos M, Thomsen J, Harmsen A: Results of translabyrinthine removal of 300 acoustic neuromas related to tumour size. Acta Otolaryngol Suppl 452:38-51, 1988

49. Tos T, Caye-Thomasen P, Stangerup SE, Tos M, Thomsen J: Long-term socio-economic impact of vestibular schwannoma for patients under observation and after surgery. J Laryngol Otol 117:955-964, 2003

50. van de Langenberg R, Hanssens PE, van Overbeeke JJ, Verheul JB, Nelemans PJ, de Bondt BJ, et al: Management of large vestibular schwannoma. Part I. Planned subtotal resection followed by Gamma Knife surgery: radiological and clinical aspects. Clinical article. J Neurosurg 115:875-884, 2011

51. Wiet RJ, Mamikoglu B, Odom L, Hoistad DL: Long-term results of the first 500 cases of acoustic neuroma surgery. Otolaryngol Head Neck Surg 124:645-651, 2001 


\section{Gamma Knife surgery for large vestibular schwannomas}

52. Wowra B, Muacevic A, Jess-Hempen A, Hempel JM, Müller-Schunk S, Tonn JC: Outpatient gamma knife surgery for vestibular schwannoma: definition of the therapeutic profile based on a 10-year experience. J Neurosurg 102 Suppl:114-118, 2005

53. Yamakami I, Uchino Y, Kobayashi E, Yamaura A, Oka N: Removal of large acoustic neurinomas (vestibular schwannomas) by the retrosigmoid approach with no mortality and minimal morbidity. J Neurol Neurosurg Psychiatry 75:453-458, 2004

54. Yang HC, Kano H, Awan NR, Lunsford LD, Niranjan A, Flickinger JC, et al: Gamma Knife radiosurgery for largervolume vestibular schwannomas. Clinical article. J Neurosurg 114:801-807, 2011

55. Yang SY, Kim DG, Chung HT, Park SH, Paek SH, Jung HW: Evaluation of tumour response after gamma knife radiosurgery for residual vestibular schwannomas based on MRI morphological features. J Neurol Neurosurg Psychiatry 79: 431-436, 2008

56. Yates PD, Jackler RK, Satar B, Pitts LH, Oghalai JS: Is it worthwhile to attempt hearing preservation in larger acoustic neuromas? Otol Neurotol 24:460-464, 2003

57. Yu CP, Cheung JY, Leung S, Ho R: Sequential volume mapping for confirmation of negative growth in vestibular schwannomas treated by gamma knife radiosurgery. J Neurosurg 93 (Suppl 3):82-89, 2000

58. Zhang X, Fei Z, Chen YJ, Fu LA, Zhang JN, Liu WP, et al: Facial nerve function after excision of large acoustic neuromas via the suboccipital retrosigmoid approach. J Clin Neurosci 12:405-408, 2005

Manuscript submitted April 7, 2012.

Accepted June 5, 2012.

Please include this information when citing this paper: DOI: 10.3171/2012.6.GKS12697.

Address correspondence to: Dar-Yu Yang, M.D., Ph.D., Department of Neurosurgery, Chang Bing Show Chwan Memorial Hospital, No. 6 Lu-Kong Road, Changhua, 505 Taiwan, R.O.C. email: yang250h@gmail.com.tw. 